\title{
Impact of Time-Correlated Arrivals on the Performance of Backpressure-Based Stochastic Network Control
}

Jeongho Jeon and Anthony Ephremides

Abstract. In this paper, we consider backpressure-based control for wireless multihop networks with time-correlated arrivals. The arrival process considered in this work is fairly general in the sense that it may exhibit short- or long-range dependence depending on the asymptotic shape of the autocorrelation function. We first show that the original backpressure policy stabilizes the network whenever the arrival rate vector is inside the stability region no matter whether the arrivals are i.i.d. or time-correlated but with monotonically decreasing autocorrelation functions. Apart from stability, the effect of correlations appears in the upper bound on average network delay. After that, we move to the case that the arrival rate vector is possibly outside the stability region. To deal with such a case, we take the method of joint flow control and backpressure policy that is known for i.i.d. arrivals to perform arbitrarily close to the utility-optimal throughput point with a corresponding tradeoff in average network delay. The tradeoff is redefined for the network with time-correlated arrivals.

(C) Taylor \& Francis Group, LLC

ISSN: 1542-795I print 


\section{Introduction}

The stability region of constrained queuing systems was introduced in [Tassiulas and Ephremides 92]; it is defined as the set of arrival rate vectors for which the queues in the network reach their steady states. A queuing system is constrained in the sense that the servers are interdependent, such as in the activation of links in a wireless network. A maximum throughput policy that supports the entire stability region except at most at points on the boundary was proposed in that paper as well. In other words, the stability of the system is achieved by the discovered policy whenever the arrival rate vector is strictly inside the stability region, which is often called the throughput optimality. The maximum throughput policy consists of two parts: maximum differential backlog routing and max-weight scheduling. Since the policy selects paths dynamically according to the differential backlog between neighboring nodes (rather than requiring them to be specified in advance), it is often referred to as backpressure policy. After the original work of [Tassiulas and Ephremides 92], there has been considerable effort on its generalization and extension [Neely et al. 05b, Georgiadis et al. 06, Neely et al. 05a, Neely 06a, Neely 06b, Neely 06c, Le et al. 10, Mekkittikul and McKeown 98]. A notable achievement concerned the case in which the arrival rate vector is possibly outside the stability region [Neely et al. 05a, Georgiadis et al. 06]. This was accomplished by augmenting the backpressure policy with a flow control mechanism that is designed to maximize network utility [Kelly 97, Kelly et al. 98, Low and Lapsley 99, Chiang 05, Mo and Walrand 00].

Despite the significance of the original work [Tassiulas and Ephremides 92] and its extensions [Neely et al. 05b, Georgiadis et al. 06, Neely et al. 05a, Neely 06a, Neely 06b, Neely 06c, Le et al. 10, Mekkittikul and McKeown 98] to stochastic network control, one weakness might be the fact that the results were mostly derived under the assumption that each arrival process has an independent and identically distributed (i.i.d.) number of arrivals in each time slot. Several measurement-based studies have pointed out, however, that scale-invariant burstiness, i.e., self-similarity, exists in local- and wide-area networks and Internet traffic [Leland et al. 94, Paxson and Floyd 95, Crovella and Bestavros 97]. A possible explanation includes heavy-tailed file-size distribution, human interaction, and protocol-level dynamics. It was also shown that variable-bit-rate video traffic is long-range dependent [Beran et al. 95]. Self-similarity and long-range dependence are two distinct concepts, and hence one does not necessarily imply the other. However, when both are viewed at asymptotically large scale, they are identical [Samorodnitsky 07].

The performance of backpressure-based stochastic network control in multihop wireless networks with time-correlated arrivals is studied in this paper using the 
Lyapunov drift technique [Tassiulas and Ephremides 92, Neely et al. 05b, Georgiadis et al. 06, Neely et al. 05a]. The arrival process considered in this work is fairly general in the sense that it may exhibit short- or long-range dependence depending on the asymptotic shape of the autocorrelation function. The cases in which the arrival rate vector is strictly inside and possibly outside the stability region are considered, and the performance of the backpressure-based policy is derived separately for both cases, incorporating statistical information of the arrival processes. To the best of our knowledge, there is no existing work that takes arrivals that are possibly long-range dependent into the analysis of backpressure-based control for general multihop networks. In [Neely et al. 05b], it was shown that the original backpressure policy [Tassiulas and Ephremides 92] is still throughput optimal with non-i.i.d. behavior of the system including arrivals and channel states. However, the work considered only the case of an arrival rate vector that is strictly inside the stability region, and the derived results do not explicitly contain statistical information of such non-i.i.d. processes, since they use the fact that the correlations will be eventually averaged out over a sufficiently long interval. In [Le et al. 09], delay performance of max-weight scheduling was studied for both single-hop and multihop networks with two-state Markov modulated arrival processes that are certainly short-range dependent. The arrival process considered in [Neely 09] is as general as ours but was considered in the context of maximal scheduling for single-hop networks.

This paper begins with the case in which the arrival rate vector is strictly inside the stability region. In this case, throughput of each flow is equal to its arrival rate by stabilization of the network. We show that the network is stabilized by the backpressure policy no matter whether the arrivals are i.i.d. or timecorrelated. The effect of correlations on the arrivals is quantified in terms of average network delay in comparison with the i.i.d. arrivals. After that, the arrival rate vector is allowed to be possibly outside the stability region, and we use the method of joint flow control and backpressure policy developed in [Neely et al. 05a, Georgiadis et al. 06]. The joint policy performs arbitrarily close to the utility-optimal throughput point with a corresponding tradeoff in average network delay. The effect of correlations on the arrival processes appears explicitly in the tradeoff, and we make a comparison with that obtained under i.i.d. arrivals.

The rest of the paper is organized as follows. In Section 2, we provide detailed descriptions of the network model and time-correlated arrivals. In Section 3, we consider the case that the arrival rate vector is inside the stability region and present a bound on average network delay under the original backpressure policy. In Section 4, the case with arbitrary arrival rate vector is considered, and the tradeoff between network utility and average delay is derived under the joint flow 


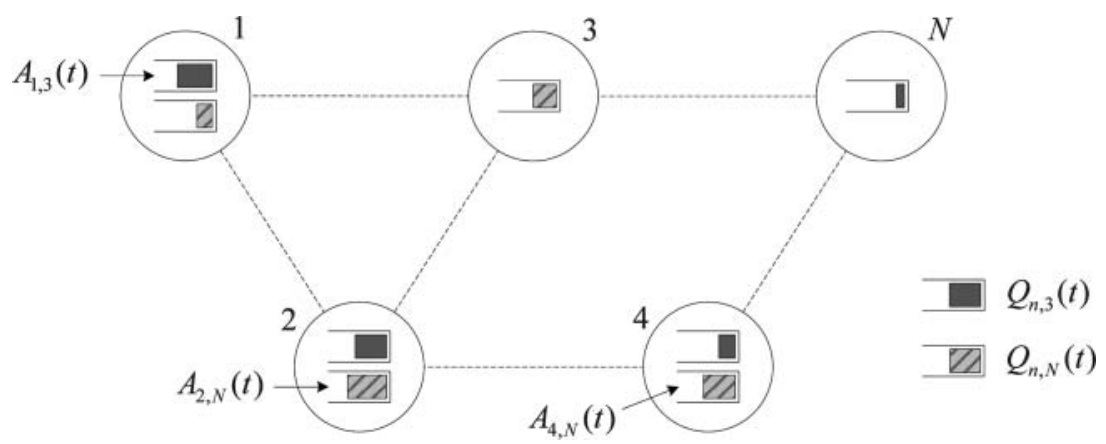

Figure I. Wireless multihop network with multiple input streams and perdestination queuing.

control and backpressure policy. In both sections, the time-correlated arrivals are modeled through processes with finite- or infinite-length memory, and the results are derived separately for each case and compared with each other. Finally, we draw conclusions in Section 5.

\section{Network Model}

We consider a time-slotted multihop wireless network with $N$ nodes and $L$ directed links. An example network topology is shown in Figure 1. We denote by $\mathcal{N}$ and $\mathcal{L}$ the sets of nodes and links, respectively. Let $A_{n, c}(t)$ represent the exogenously arriving amount of data at node $n$ during time slot $t$ that is destined to node $c$ in units of bits/slot. At each node, all the exogenous and endogenous arrivals due to multihop relaying are classified and queued according to their destinations. We let $Q_{n, c}(t)$ be the backlog of destination- $c$ data that is awaiting transmission in node $n$ at time slot $t$ in units of bits, and define $\vec{Q}(t) \triangleq\left(Q_{n, c}(t)\right)$. It is assumed that the system starts with empty queues, i.e., $Q_{n, c}(0)=0$ for all $(n, c)$ pairs. Denote by $\mu_{l}(t)$ and $\mu_{l, c}(t)$ the transmission rate over link $l$ during time slot $t$ and the amount of rate that is offered to destination- $c$ traffic among $\mu_{l}(t)$ in units of bits/slot, respectively. Consequently, $\sum_{c} \mu_{l, c}(t) \leq \mu_{l}(t)$ for all $l$. The network is constrained because the transmission rate of each link depends not only on the time-varying channel state but also on the activation of the other links. Denote by $\Gamma_{t}$ the feasible region of link transmission rate vector $\vec{\mu}(t) \triangleq\left(\mu_{l}(t)\right)$ at time slot $t$. In each time slot, a control policy chooses $\vec{\mu}(t)$ from the constrained set $\Gamma_{t}$ and allocates the rate on each link to the traffic of each destination. 
In this work, the arrival process $A_{n, c}(t)$ does not necessarily have to be i.i.d. but need only be wide-sense stationary (WSS), that is, a process with constant mean $\lambda_{n, c}$, finite variance $\sigma_{n, c}^{2}$, and an autocorrelation function $\rho_{n, c}(k) \triangleq$ $E\left[\left(A_{n, c}(t)-\lambda_{n, c}\right)\left(A_{n, c}(t-k)-\lambda_{n, c}\right)\right] / \sigma_{n, c}^{2}$ that depends only on the time lag between two samples. Denote by $H(t)$ the past history of all arrivals up to but not including time slot $t$. If there exists a finite number $T \geq 0$ such that $E\left[A_{n, c}(t) \mid H(t-k)\right]=\lambda_{n, c}$ for $k \geq T$, then $\rho_{n, c}(k)=0$ for $k>T$. Stochastic processes that fall in this category are said to have finite-length memory; an i.i.d. process is a special case with $T=0$. If there is no such finite $T$, we further divide the processes according to the asymptotic shape of the autocorrelation function $\rho_{n, c}(k)$. For most of the stochastic models including autoregressive moving average processes and Markov modulated processes, the autocorrelation functions are characterized by an exponential decay, i.e., $\rho_{n, c}(k) \sim \alpha^{k}$ as $k \rightarrow \infty$, where $0<\alpha<1$. The exponential tail of the function implies $\sum_{k} \rho_{n, c}(k)<\infty$. Stochastic processes belonging to this category are said to be short-range dependent. The burstiness of the input traffic modeled by a short-range dependent process would tend to be smoothed by averaging over a long enough time scale. On the other hand, long-range dependent processes are characterized by a power-law decay of autocorrelation function, $\rho_{n, c}(k) \sim k^{-\beta}$ as $k \rightarrow \infty$, where $0<\beta<1$. As a result, the autocorrelation function is nonsummable, i.e., $\sum_{k} \rho_{n, c}(k)=\infty$, which implies that while high-lag correlations are individually small, their cumulative effect gives rise to features that are drastically different from those of shortrange dependent processes [Beran et al. 95]. Examples of long-range dependent processes are fractional Brownian motion and its discrete-time analogue, fractional Gaussian noise. The degree of self-similarity of a series is expressed using a single parameter $H$, called the Hurst parameter. Self-similar processes and longrange dependent processes are related through the relation $H=1-\beta / 2$; for a self-similar process with long-range dependence, we have $1 / 2<H<1$. Nevertheless, these are two different concepts: self-similarity involves all scales, whereas long-range dependence involves only an asymptotically large scale. Yet, if we are interested only in steady-state performance measures such as average network delay, the role of self-similar input traffic is not different from that of long-range dependent traffic.

\section{Network with Stabilizable Arrival Rates}

Define $\mathcal{I}_{n}$ and $\mathcal{O}_{n}$ as the set of incoming and outgoing links of node $n$, i.e., $\{l: \operatorname{rx}(l)=n\}$ and $\{l: \operatorname{tx}(l)=n\}$, where $\operatorname{tx}(l)$ and $\operatorname{rx}(l)$ are the transmitting and receiving nodes of link $l$, respectively. Then the queue length process at 


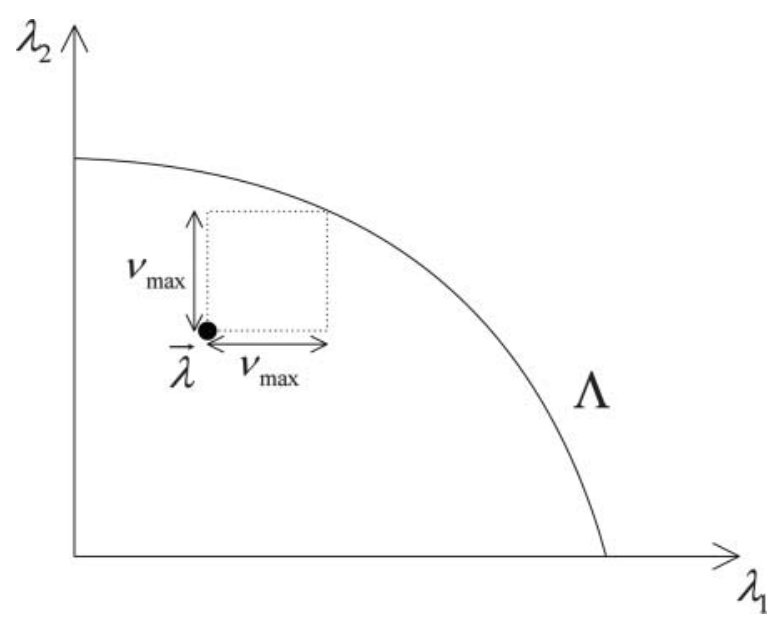

Figure 2. Illustration of two-dimensional stability region with arrival rate vector inside the region.

node $n$ for destination- $c$ data evolves as

$$
Q_{n, c}(t+1) \leq \max \left[Q_{n, c}(t)-\sum_{l \in \mathcal{O}_{n}} \mu_{l, c}(t), 0\right]+\sum_{l \in \mathcal{I}_{n}} \mu_{l, c}(t)+A_{n, c}(t),
$$

which is an inequality rather than an equality because the actual endogenous arrivals may be less than the allocated link rates if the corresponding transmitters do not have enough data. The reason for (3.1) to contain the max operation is the same. The stability region $\Lambda$ of a system is defined as the set of arrival rate vectors $\vec{\lambda} \triangleq\left(\lambda_{n, c}\right)$ for which all queues in the network are stable by considering all the policies [Tassiulas and Ephremides 92]. ${ }^{1}$ Assuming that the arrival rate vector is inside the stability region, i.e., $\vec{\lambda} \in \Lambda$, as shown in Figure 2, the exact throughput equal to the arrival rate is achieved at each flow simply by stabilizing the network. It is well known that the backpressure policy given in Algorithm 1 stabilizes a network if the arrival rate vector is inside the stability region and each arrival process has i.i.d. number of arrivals in each time slot [Tassiulas and Ephremides 92, Georgiadis et al. 06].

Note that the policy lets the maximizing destination- $c^{*}$ data use all the rate allocated to the link. Therefore, in each time slot, $\mu_{l, c^{*}}(t)=\mu_{l}(t)$ and $\mu_{l, c}(t)=0$ for all $c \neq c^{*}$.

Before we present the performance of the backpressure policy with timecorrelated arrivals, let us first describe the following constants. Denote by $A_{\max }$

\footnotetext{
${ }^{1} \mathrm{~A}$ queue is said to be stable if it reaches a steady state and does not drift to infinity.
} 


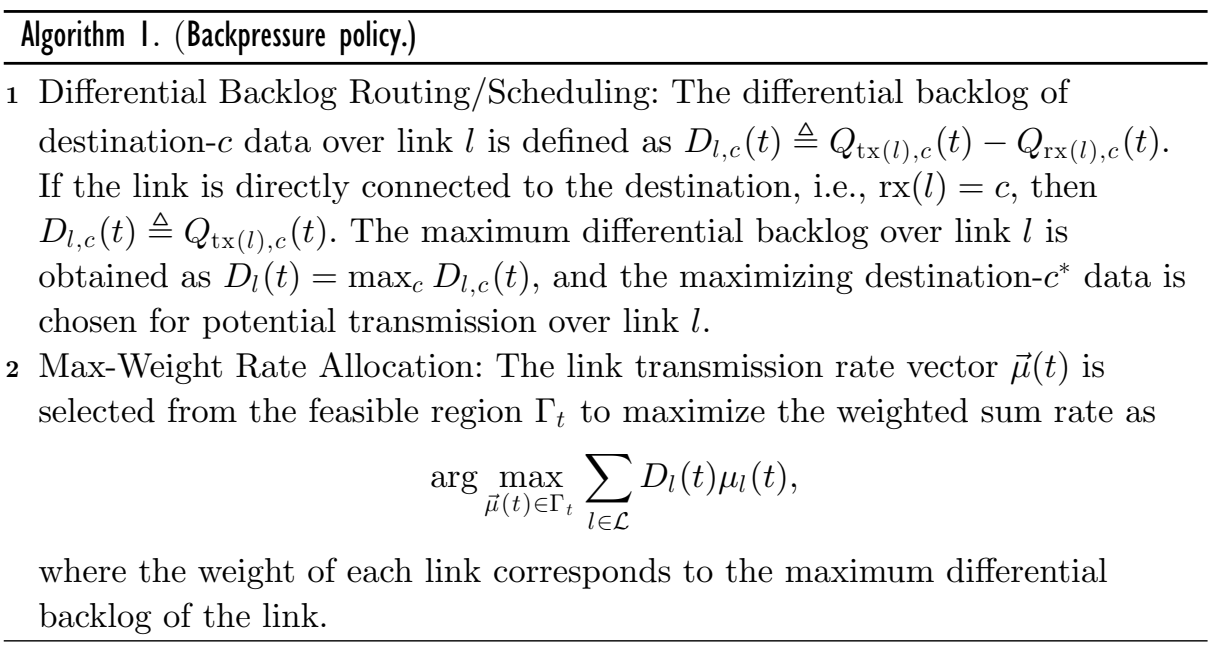

the maximum amount of arrivals to any node in any slot, i.e., $\sum_{c} A_{n, c}(t) \leq A_{\max }$. Further define $\mu_{\max }^{\text {out }}$ and $\mu_{\max }^{\text {in }}$ as the maximum transmission rates out of, and into, any node for all time slots as $\sum_{l \in \mathcal{O}_{n}} \mu_{l}(t) \leq \mu_{\max }^{\text {out }}$ and $\sum_{l \in \mathcal{I}_{n}} \mu_{l}(t) \leq \mu_{\max }^{\text {in }}$, respectively.

Theorem 3.I. If the arrival rate vector $\vec{\lambda}$ is strictly interior to the stability region $\Lambda$ and there exists a finite integer $T>0$ such that $E\left[A_{n, c}(t) \mid H(t-k)\right]=\lambda_{n, c}$ for $k \geq T$ and for all $(n, c)$ pairs, the backpressure policy stabilizes the network and guarantees a bounded average queue backlog as

$$
\limsup _{t \rightarrow \infty} \frac{1}{t} \sum_{\tau=0}^{t-1} \sum_{n, c} E\left[Q_{n, c}(\tau)\right] \leq \frac{1}{2 \nu_{\max }}\left(N B_{1}+2 \sum_{n, c} \phi_{n, c}(T)\right),
$$

where

$$
\phi_{n, c}(T) \triangleq \sigma_{n, c}^{2} \sum_{k=1}^{T} \rho_{n, c}(k)+T \lambda_{n, c}\left(\mu_{\max }^{\text {in }}+\lambda_{n, c}\right)
$$

and $B_{1} \triangleq\left(\mu_{\max }^{\mathrm{in}}+A_{\max }\right)^{2}+\left(\mu_{\max }^{\text {out }}\right)^{2}$, and $\nu_{\max }$ is defined as the maximum of $\nu$ such that $\vec{\lambda}+\nu \overrightarrow{1} \in \Lambda$, where $\overrightarrow{1}$ is a vector whose cardinality is the same as that of $\vec{\lambda}$ and whose elements are all equal to 1 .

Proof. Define $L(\vec{Q}(t)) \triangleq \sum_{n, c} Q_{n, c}(t)^{2}$ as a Lyapunov function for scalar measure of the network congestion. From the queuing dynamics in (3.1), it can be verified 
that

$$
\begin{aligned}
Q_{n, c}(t+1)^{2} \leq & Q_{n, c}(t)^{2}+\left(\sum_{l \in \mathcal{O}_{n}} \mu_{l, c}(t)\right)^{2}+\left(\sum_{l \in \mathcal{I}_{n}} \mu_{l, c}(t)+A_{n, c}(t)\right)^{2} \\
& -2 Q_{n, c}(t)\left(\sum_{l \in \mathcal{O}_{n}} \mu_{l, c}(t)-\sum_{l \in \mathcal{I}_{n}} \mu_{l, c}(t)\right)+2 Q_{n, c}(t) A_{n, c}(t) .
\end{aligned}
$$

Summing over all $(n, c)$ pairs and taking the conditional expectation given the current queue backlog $\vec{Q}(t)$ yields the conditional Lyapunov drift satisfying

$$
\begin{aligned}
\Delta(\vec{Q}(t)) \triangleq & E[L(\vec{Q}(t+1))-L(\vec{Q}(t)) \mid \vec{Q}(t)] \\
\leq & N B_{1}-2 \sum_{n, c} Q_{n, c}(t) E\left[\sum_{l \in \mathcal{O}_{n}} \mu_{l, c}(t)-\sum_{l \in \mathcal{I}_{n}} \mu_{l, c}(t) \mid \vec{Q}(t)\right] \\
& +2 \sum_{n, c} Q_{n, c}(t) E\left[A_{n, c}(t) \mid \vec{Q}(t)\right] .
\end{aligned}
$$

Note that the control variables on the right-hand side of the drift expression are link transmission rates $\mu_{l, c}(t)$. By simply converting the corresponding terms containing control variables from node-centric to link-centric expressions, we obtain the following identity:

$$
\sum_{n, c} Q_{n, c}(t)\left(\sum_{l \in \mathcal{O}_{n}} \mu_{l, c}(t)-\sum_{l \in \mathcal{I}_{n}} \mu_{l, c}(t)\right)=\sum_{l} \sum_{c} \mu_{l, c}(t)\left(Q_{\operatorname{tx}(l), c}(t)-Q_{\operatorname{rx}(l), c}(t)\right),
$$

which reveals the rationale behind the design of the backpressure policy; it is designed to minimize the right-hand side of (3.2) by maximizing (3.3). The original proof in [Tassiulas and Ephremides 92] showed that for a sufficiently large sum of queue backlogs, the right-hand side of (3.2) under the backpressure policy becomes negative, which corresponds to Forster's criteria for stability of irreducible Markov chains [Asmussen 03]. In [Neely et al. 05b, Georgiadis et al. 06, Neely et al. 05a], the Lyapunov drift technique is extended so that an explicit upper bound on the sum of average queue backlogs can be obtained that suffices for the stability proof as well. We follow similar procedures here. Note that because we are considering the case $\vec{\lambda} \in \Lambda$, there must exist some constant $\nu>0$ such that $\vec{\lambda}+\nu \overrightarrow{1} \in \Lambda$. From [Georgiadis et al. 06, Corollary 3.9], we know that there exists a stationary randomized policy that makes decisions based only on the current channel states and independently of queue backlogs such that

$$
E\left[\sum_{l \in \mathcal{O}_{n}} \mu_{l, c}(t)-\sum_{l \in \mathcal{I}_{n}} \mu_{l, c}(t) \mid \vec{Q}(t)\right]=\lambda_{n, c}+\nu
$$


Since the stationary policy is simply a particular rate allocation policy, the performance under the backpressure policy is no worse than any other policies following the construction of the algorithm. Consequently, we have

$$
\Delta(\vec{Q}(t)) \leq N B_{1}-2 \sum_{n, c}\left(\lambda_{n, c}+\nu\right) Q_{n, c}(t)+2 \sum_{n, c} Q_{n, c}(t) E\left[A_{n, c}(t) \mid \vec{Q}(t)\right] .
$$

In the previous works assuming i.i.d. arrivals, the relation $E\left[A_{n, c}(t) \mid \vec{Q}(t)\right]=\lambda_{n, c}$ holds, which simplifies the rest of the analysis. However, if arrivals are correlated, then $Q_{n, c}(t)$ is also correlated through the queuing dynamics in (3.1), and $A_{n, c}(t)$ and $Q_{n, c}(t)$ are no longer independent of each other. The multislot Lyapunov drift technique proposed in [Tassiulas 97] and elaborated in [Neely et al. 05b] can be envisioned; since the technique uses the fact that the non-i.i.d. behavior of the system would be eventually averaged out over a sufficiently long interval, the results obtained using this technique do not explicitly contain the correlation structure of the arrival process. Therefore, we need a somewhat direct approach as follows. We note from the queuing dynamics that

$$
Q_{n, c}(t) \leq Q_{n, c}(t-T)+\sum_{k=1}^{T}\left(\sum_{l \in \mathcal{I}_{n}} \mu_{l, c}(t-k)+A_{n, c}(t-k)\right),
$$

and thus it follows that

$$
\begin{aligned}
E\left[Q_{n, c}(t) A_{n, c}(t)\right] \leq & E\left[Q_{n, c}(t-T) A_{n, c}(t)\right] \\
& +E\left[\sum_{k=1}^{T}\left(\sum_{l \in \mathcal{I}_{n}} \mu_{l, c}(t-k)+A_{n, c}(t-k)\right) A_{n, c}(t)\right] .
\end{aligned}
$$

Because all the arrival processes are assumed to have finite memory of length at most $T$, we have

$$
\begin{aligned}
E\left[Q_{n, c}(t-T) A_{n, c}(t)\right] & =E\left[Q_{n, c}(t-T) E\left[A_{n, c}(t) \mid \vec{Q}(t-T)\right]\right] \\
& =\lambda_{n, c} E\left[Q_{n, c}(t-T)\right] .
\end{aligned}
$$

For the remainder of the right-hand side of (3.5), we obtain

$$
\begin{gathered}
E\left[\sum_{k=1}^{T}\left(\sum_{l \in \mathcal{I}_{n}} \mu_{l, c}(t-k)+A_{n, c}(t-k)\right) A_{n, c}(t)\right] \\
\leq \sigma_{n, c}^{2} \sum_{k=1}^{T} \rho_{n, c}(k)+T \lambda_{n, c}\left(\mu_{\max }^{\text {in }}+\lambda_{n, c}\right) .
\end{gathered}
$$

Plugging (3.6) and (3.7) into the right-hand side of (3.5) yields an upper bound on $E\left[Q_{n, c}(t) A_{n, c}(t)\right]$. Taking the expectation of (3.4) with respect to the 
distribution of queue backlogs and applying the bound yields an unconditional Lyapunov drift satisfying

$$
\begin{aligned}
& E[L(\vec{Q}(t+1))-L(\vec{Q}(t))] \leq N B_{1}-2 \sum_{n, c}\left(\lambda_{n, c}+\nu\right) E\left[Q_{n, c}(t)\right] \\
& \quad+2 \sum_{n, c} \lambda_{n, c} E\left[Q_{n, c}(t-T)\right]+2 \sum_{n, c}\left(\sigma_{n, c}^{2} \sum_{k=1}^{T} \rho_{n, c}(k)+T \lambda_{n, c}\left(\mu_{\max }^{\text {in }}+\lambda_{n, c}\right)\right) .
\end{aligned}
$$

Summing the inequality over $t \in\{0, \ldots, M-1\}$ yields

$$
\begin{aligned}
& E[L(\vec{Q}(M))-L(\vec{Q}(0))] \\
& \leq N M B_{1}-2 \sum_{\tau=0}^{M-1} \sum_{n, c}\left(\lambda_{n, c}+\nu\right) E\left[Q_{n, c}(\tau)\right]+2 \sum_{\tau=0}^{M-1} \sum_{n, c} \lambda_{n, c} E\left[Q_{n, c}(\tau-T)\right] \\
& \quad+2 M \sum_{n, c}\left(\sigma_{n, c}^{2} \sum_{k=1}^{T} \rho_{n, c}(k)+T \lambda_{n, c}\left(\mu_{\max }^{\text {in }}+\lambda_{n, c}\right)\right)
\end{aligned}
$$

where $E\left[Q_{n, c}(t)\right]=0$ for $t \leq 0$. Dividing the above by $2 M$, rearranging terms, and using the fact that the system starts with empty queues and the nonnegativity of the Lyapunov function, we obtain

$$
\begin{aligned}
& \frac{1}{M} \sum_{\tau=0}^{M-1} \sum_{n, c}\left(\lambda_{n, c}+\nu\right) E\left[Q_{n, c}(\tau)\right]-\frac{1}{M} \sum_{\tau=0}^{M-1} \sum_{n, c} \lambda_{n, c} E\left[Q_{n, c}(\tau-T)\right] \\
& \quad \leq \frac{N B_{1}}{2}+\sum_{n, c}\left(\sigma_{n, c}^{2} \sum_{k=1}^{T} \rho_{n, c}(k)+T \lambda_{n, c}\left(\mu_{\max }^{\text {in }}+\lambda_{n, c}\right)\right) .
\end{aligned}
$$

Taking the $\lim \sup$ as $M \rightarrow \infty$, noting that

$$
\limsup _{M \rightarrow \infty} \frac{1}{M} \sum_{\tau=0}^{M-1} E\left[Q_{n, c}(\tau-T)\right]=\limsup _{M \rightarrow \infty} \frac{1}{M} \sum_{\tau=0}^{M-1} E\left[Q_{n, c}(\tau)\right],
$$

and optimizing over $\nu$ yields the result.

Theorem 3.1 guarantees the stability of the network under the backpressure policy when the arrival rate vector is inside the stability region and the arrival processes are time-correlated over a fixed length of interval. This is done by explicitly showing that the sum of average queue backlogs in the network is bounded above by some finite number. However, if there is at least one long memory arrival such that the condition $E\left[A_{n, c}(t) \mid H(t-k)\right]=\lambda_{n, c}$ for $k \geq T$ is invalidated for any finite integer $T$, the theorem fails simply because the upper bound becomes infinite. The arrivals with short-range dependence might be dealt with similarly to the arrivals with finite memory using the fact that their autocorrelations decrease exponentially fast as the time-lag increases. The following 
theorem, on the other hand, is applicable for both short-range and long-range dependent arrivals, since it requires only very mild conditions on the arrival process such as the monotonicity of its absolute autocorrelation function.

Theorem 3.2. If arrival rate vector $\vec{\lambda}$ is strictly interior to the stability region $\Lambda$ and the absolute autocorrelation functions $\left|\rho_{n c}(k)\right|$ of the arrival processes are monotonically decreasing for all $(n, c)$ pairs, the backpressure policy stabilizes the network and guarantees bounded average queue backlog as

$$
\limsup _{t \rightarrow \infty} \frac{1}{t} \sum_{\tau=0}^{t-1} \sum_{n, c} E\left[Q_{n, c}(\tau)\right] \leq \frac{1}{2\left(\nu_{\max }-\delta\right)}\left(N B_{1}+2 \sum_{n, c} \phi_{n, c}\left(T_{\delta}\right)\right),
$$

where $\delta$ is an arbitrary constant satisfying $0<\delta<\nu_{\max }$ and $T_{\delta}$ is the minimum of $T$ such that $\left|E\left[A_{n, c}(t) \mid H(t-k)\right]-\lambda_{n, c}\right| \leq \delta$ for $k \geq T$ and for all $(n, c)$ pairs.

Proof. As in the proof of Theorem 3.1, the main difficulty in analyzing the Lyapunov drift is due to the correlation between $Q_{n, c}(t)$ and $A_{n, c}(t)$. Assume that $\delta$ and corresponding $T_{\delta}$ are chosen such that the conditions described in the theorem are met. Then for $T \geq T_{\delta}$, we have

$$
\begin{aligned}
E\left[Q_{n, c}(t-T) A_{n, c}(t)\right] & =E\left[Q_{n, c}(t-T) E\left[A_{n, c}(t) \mid \vec{Q}(t-T)\right]\right] \\
& \leq\left(\lambda_{n, c}+\delta\right) E\left[Q_{n, c}(t-T)\right]
\end{aligned}
$$

for all $(n, c)$ pairs, and inequality (3.7) holds for every $T>0$. Therefore, the unconditional Lyapunov drift satisfies

$$
\begin{aligned}
& E[L(\vec{Q}(t+1))-L(\vec{Q}(t))] \\
& \leq N B_{1}-2 \sum_{n, c}\left(\lambda_{n, c}+\nu\right) E\left[Q_{n, c}(t)\right]+2 \sum_{n, c}\left(\lambda_{n, c}+\delta\right) E\left[Q_{n, c}(t-T)\right] \\
& \quad+2 \sum_{n, c}\left(\sigma_{n, c}^{2} \sum_{k=1}^{T} \rho_{n, c}(k)+T \lambda_{n, c}\left(\mu_{\max }^{\text {in }}+\lambda_{n, c}\right)\right)
\end{aligned}
$$

for $T \geq T_{\delta}$. The rest of the proof is identical to that of Theorem 3.1.

Theorem 3.2 can be applied to the network containing any long memory arrivals but with absolute autocorrelation functions that are monotonically decreasing. It is expected that long-range dependent arrivals would induce longer $T_{\delta}$ satisfying the condition when compared to short-range dependent arrivals due to the hyperbolic shape of the autocorrelation function. Once the autocorrelation functions are given, one can compute $T_{\delta}$ deterministically. From Theorems 3.1 and 3.2, we know that the backpressure policy stabilizes the network whenever the arrival rate vector is inside the stability region no matter whether the arrival processes are i.i.d. or time-correlated. Specifically, in Theorem 3.1, the upper 
bound on the sum of average queue backlogs is always finite whenever $\nu_{\max }>0$, which corresponds to the condition that the arrival rate vector is strictly inside the stability region. In Theorem 3.2, there must exist $\delta$ such that $0<\delta<\nu_{\max }$ because $\nu_{\max }>0$ and $T_{\delta}$ must be finite given $\delta$, because the autocorrelation functions are assumed to be monotonically decreasing. Therefore, the upper bound on the sum of average queue backlogs is always finite. Note that in case of i.i.d. arrivals, the upper bound is given by $N B_{1} / 2 \nu_{\max }$ [Georgiadis et al. 06]. Thus, apart from the stability issue, the upper bound on average network delay will increase when arrivals are time-correlated, because the average network delay is proportional to the average network backlog by Little's law [Bertsekas and Gallager 92].

\section{Network with Arbitrary Arrival Rates}

In this section, we consider an arrival rate vector $\vec{\lambda}$ that is possibly outside the stability region $\Lambda$, as shown in Figure 3 . If $\vec{\lambda} \notin \Lambda$, the network will certainly be unstable with any policy unless we have a set of flow controllers in front of queues as shown in Figure 4 . Denote by $R_{n, c}(t)$ the amount of data that is admitted into the network by the flow controller at time slot $t$ and define $r_{n, c} \triangleq$ $\lim _{t \rightarrow \infty} \frac{1}{t} \sum_{\tau=0}^{t-1} E\left[R_{n, c}(\tau)\right]$. The role of flow controllers is to choose $R_{n, c}(t)(\leq$ $\left.A_{n, c}(t)\right)$ in each time slot such that the average admission rate vector $\vec{r}=\left(r_{n, c}\right)$ lies inside the stability region. In that case, the stability of the network can be achieved by the backpressure policy in Algorithm 1 by stabilizing queues in the

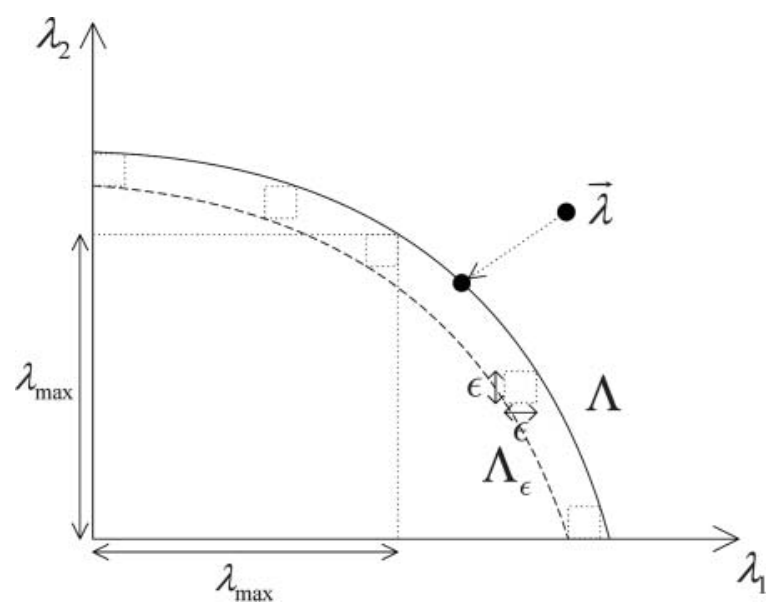

Figure 3. Illustration of $\epsilon$-stripped stability region $\Lambda_{\epsilon}$ with arrival rate vector outside the stability region $\Lambda$. 


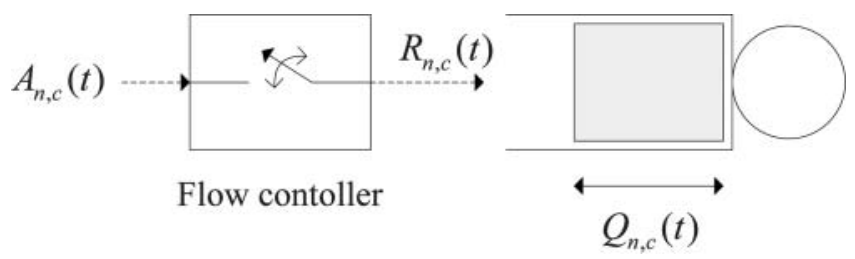

Figure 4. A queuing model with flow controller.

network that are now evolving as

$$
Q_{n, c}(t+1) \leq \max \left[Q_{n, c}(t)-\sum_{l \in \mathcal{O}_{n}} \mu_{l, c}(t), 0\right]+\sum_{l \in \mathcal{I}_{n}} \mu_{l, c}(t)+R_{n, c}(t),
$$

where the exogenous arrival $A_{n, c}(t)$ is replaced by $R_{n, c}(t)$ from (3.1).

The flow controllers need to be designed in some optimum way that is equivalent to the question of which point on the stability region $\Lambda$ is to be achieved. This is usually done by assigning a utility to each flow that is a measure of relative satisfaction and maximizing the total network utility as in [Kelly 97, Kelly et al. 98, Low and Lapsley 99, Chiang 05, Mo and Walrand 00]:

$$
\begin{aligned}
& \text { maximize } \sum_{n, c} U_{n, c}\left(r_{n, c}\right) \\
& \text { subject to } \vec{r} \in \Lambda, \quad 0 \leq r_{n, c} \leq \lambda_{n, c}, \forall(n, c),
\end{aligned}
$$

where $U_{n, c}(\cdot)$ is the utility function associated with the input stream described by an $(n, c)$ pair. The first constraint in the formulation states that the average admitted rate vector must be stabilizable, and the next set of constraints state that the admission rate of each stream must not exceed its incoming rate. Those constraints are automatically met under any policy that stabilizes the network. It is widely assumed that utility functions are strictly concave, nondecreasing, and continuously differentiable on the positive real axis. As a result, the first derivative of the utility function is monotonically decreasing with maximum at $r=0$. We assume that the maximum is bounded by some finite value $\theta_{n, c} .^{2}$ It is further assumed that the aggregate utility obtained at any node for possibly multiple input streams is bounded by some finite value $U_{\max }$.

In [Georgiadis et al. 06, Neely et al. 05a], the Lyapunov drift technique was used to attain both stability and performance optimization simultaneously, and the resulting joint flow control and backpressure policy were shown for i.i.d. arrivals to come arbitrarily close to the optimal solution of (4.1) with a corresponding tradeoff in average network delay.

\footnotetext{
${ }^{2}$ For example, $U_{n, c}(r)=\log (1+r)$ satisfies the assumption but not $U_{n, c}(r)=\log (r)$, which is a matter of choice.
} 
Theorem 4.I. If there exists a finite integer $T>0$ such that $E\left[A_{n, c}(t) \mid H(t-k)\right]=$ $\lambda_{n, c}$ for $k \geq T$ and for all $(n, c)$ pairs, then the joint flow control and backpressure policy stabilizes the network and yields bounds on the time average queue backlog as

$$
\limsup _{t \rightarrow \infty} \frac{1}{t} \sum_{\tau=0}^{t-1} \sum_{n, c} E\left[Q_{n, c}(\tau)\right] \leq \frac{1}{2 \lambda_{\max }}\left(N B_{2}+V N U_{\max }+2 \sum_{n, c} \psi_{n, c}(T)\right)
$$

and network utility as

$$
\liminf _{t \rightarrow \infty} \sum_{n, c} U_{n, c}\left(\bar{r}_{n, c}(t)\right) \geq \sum_{n, c} U_{n, c}\left(r_{n, c}^{*}\right)-\frac{1}{V}\left(N B_{2}+2 \sum_{n, c} \psi_{n, c}(T)\right),
$$

where $\bar{r}_{n, c}(t) \triangleq \frac{1}{t} \sum_{\tau=0}^{t-1} E\left[R_{n, c}(\tau)\right]$ and

$$
\psi_{n, c}(T) \triangleq\left\{2 \sigma_{n, c}^{2} \sum_{k=1}^{T} \rho_{n, c}(k)+T \lambda_{n, c}\left(\mu_{\max }^{\mathrm{in}}+\lambda_{n, c}\right)\right\},
$$

and $B_{2} \triangleq B_{1}+2 A_{\max }^{2}, \lambda_{\max }$ is the largest scalar such that $\left(\lambda_{\max }\right) \in \Lambda$, and $\left(r_{n, c}^{*}\right)$ is the utility-optimal rate allocation vector.

\section{Algorithm 2. (Joint flow control and backpressure policy.)}

1 Flow Control: For every time slot and for each input stream $(n, c)$, observe $Q_{n, c}(t)$ and $Y_{n, c}(t)$ and choose

$$
R_{n, c}(t)= \begin{cases}A_{n, c}(t) & \text { if } Y_{n, c}(t) \geq Q_{n, c}(t), \\ 0, & \text { otherwise }\end{cases}
$$

where $Y_{n, c}(t)$ is updated according to

$$
Y_{n, c}(t+1)=\max \left[Y_{n, c}(t)-R_{n, c}(t), 0\right]+\gamma_{n, c}(t),
$$

which is called the virtual flow state queue, in which we set $\gamma_{n, c}(t)=\gamma_{n, c}$, where $\gamma_{n, c}$ is the solution to

$$
\begin{aligned}
& \text { maximize } V U_{n, c}\left(\gamma_{n, c}\right)-2 Y_{n, c}(t) \gamma_{n, c} \\
& \text { subject to } 0 \leq \gamma_{n, c} \leq A_{\max },
\end{aligned}
$$

where $V>0$ is a design parameter that affects the utility-delay tradeoff of the algorithm.

2 Routing/scheduling and resource allocation are performed as in Algorithm 1. 
Proof. We begin by noting that problem (4.1) is equivalent to the problem of maximizing $\sum_{n, c} U_{n, c}\left(\gamma_{n, c}\right)$ with the additional set of constraints $0 \leq \gamma_{n, c} \leq r_{n, c}$ for all $(n, c)$ pairs, because utility functions are nondecreasing. The constraints are satisfied by stabilizing the virtual flow state queues in $(4.2) \cdot{ }^{3}$ Define $L(\vec{\Theta}(t)) \triangleq$ $\sum_{n, c}\left(Q_{n, c}(t)^{2}+Y_{n, c}(t)^{2}\right)$ as a Lyapunov function whose conditional drift is described as

$$
\Delta(\vec{\Theta}(t)) \triangleq E[L(\vec{\Theta}(t+1))-L(\vec{\Theta}(t)) \mid \vec{\Theta}(t)]
$$

where $\vec{\Theta}(t) \triangleq[\vec{Q}(t) ; \vec{Y}(t)]$. With the drift computation details omitted for brevity, the conditional Lyapunov drift can be shown to satisfy

$$
\begin{aligned}
& \Delta(\vec{\Theta}(t))-V \sum_{n, c} E\left[U_{n, c}\left(\gamma_{n, c}(t)\right) \mid \vec{\Theta}(t)\right] \\
& \leq N B_{2}-2 \sum_{n, c} Q_{n, c}(t) E\left[\sum_{l \in \mathcal{O}_{n}} \mu_{l, c}(t)-\sum_{l \in \mathcal{I}_{n}} \mu_{l, c}(t) \mid \vec{\Theta}(t)\right] \\
& \quad+2 \sum_{n, c}\left(Q_{n, c}(t)-Y_{n, c}(t)\right) E\left[R_{n, c}(t) \mid \vec{\Theta}(t)\right] \\
& \quad+2 \sum_{n, c} Y_{n, c}(t) E\left[\gamma_{n, c}(t) \mid \vec{\Theta}(t)\right]-V \sum_{n, c} E\left[U_{n, c}\left(\gamma_{n, c}(t)\right) \mid \vec{\Theta}(t)\right],
\end{aligned}
$$

where the optimization metric $V \sum_{n, c} E\left[U_{n, c}\left(\gamma_{n, c}(t)\right) \mid \vec{\Theta}(t)\right]$ was subtracted on both sides. It is apparent that the joint flow control and backpressure policy in Algorithm 2 is designed to minimize the right-hand side of (4.4) among all other policies.

Define for some $\epsilon>0, \Lambda_{\epsilon} \triangleq\{\vec{r} \mid \vec{r}+\epsilon \overrightarrow{1} \in \Lambda\}$ and let $\left(r_{n, c}^{*}(\epsilon)\right)$ be the optimal solution to (4.1) with the reduced stability region $\Lambda_{\epsilon}$. From [Georgiadis et al. 06, Corollary 3.9], we know that there exists a stationary randomized policy that makes decisions based only on the current channel states and independent of queue backlogs, so that

$$
E\left[\sum_{l \in \mathcal{O}_{n}} \mu_{l, c}(t)-\sum_{l \in \mathcal{I}_{n}} \mu_{l, c}(t) \mid \vec{\Theta}(t)\right]=r_{n, c}^{*}(\epsilon)+\epsilon
$$

for all $(n, c)$ pairs. We further fix $\gamma_{n, c}(t)$ to $r_{n, c}^{*}(\epsilon)$ for all $t$ and consider a randomized policy for $R_{n, c}(t)$ such that $R_{n, c}(t)=A_{n, c}(t)$ with probability $r_{n, c}^{*}(\epsilon) / \lambda_{n, c}$ and $R_{n, c}(t)=0$ with probability $1-r_{n, c}^{*}(\epsilon) / \lambda_{n, c}$. Because the joint flow control

\footnotetext{
${ }^{3}$ The virtual queues were originally introduced in [Neely et al. 05a], and they are often used to ensure long-term average constraints.
} 
and backpressure policy in Algorithm 2 outperforms any other policy following from the construction of the algorithm, the conditional Lyapunov drift satisfies

$$
\begin{aligned}
& \Delta(\vec{\Theta}(t))-V \sum_{n, c} E\left[U_{n, c}\left(\gamma_{n, c}(t)\right) \mid \vec{\Theta}(t)\right] \\
& \leq N B_{2}-2 \sum_{n, c}\left(r_{n, c}^{*}(\epsilon)+\epsilon\right) Q_{n, c}(t) \\
& \quad+2 \sum_{n, c} \frac{r_{n, c}^{*}(\epsilon)}{\lambda_{n, c}}\left(Q_{n, c}(t)-Y_{n, c}(t)\right) E\left[A_{n, c}(t) \mid \vec{\Theta}(t)\right] \\
& \quad+2 \sum_{n, c} r_{n, c}^{*}(\epsilon) Y_{n, c}(t)-V \sum_{n, c} U_{n, c}\left(r_{n, c}^{*}(\epsilon)\right)
\end{aligned}
$$

Note that because the arrivals are time-correlated, $A_{n, c}(t)$ is not independent with $Q_{n, c}(t)$ and $Y_{n, c}(t)$ and, thus, we take a similar approach as the one used in the previous section. From the proof of Theorem 3.1, we know that

$$
E\left[Q_{n, c}(t) A_{n, c}(t)\right] \leq \lambda_{n, c} E\left[Q_{n, c}(t-T)\right]+\sigma_{n, c}^{2} \sum_{k=1}^{T} \rho_{n, c}(k)+T \lambda_{n, c}\left(\mu_{\max }^{\mathrm{in}}+\lambda_{n, c}\right) .
$$

Furthermore, from (4.2), we note that

$$
Y_{n, c}(t) \geq Y_{n, c}(t-T)-\sum_{k=1}^{T} R_{n, c}(t-k)+\sum_{k=1}^{T} \gamma_{n, c}(t-k),
$$

and thus it follows that

$$
\begin{aligned}
& E\left[Y_{n, c}(t) A_{n, c}(t)\right] \\
& \geq E\left[Y_{n, c}(t-T) A_{n, c}(t)\right]-E\left[\sum_{k=1}^{T} R_{n, c}(t-k) A_{n, c}(t)\right] \\
& \quad+E\left[\sum_{k=1}^{T} \gamma_{n, c}(t-k) A_{n, c}(t)\right] \\
& \quad \lambda_{n, c} E\left[Y_{n, c}(t-T)\right]-\frac{r_{n, c}^{*}(\epsilon)}{\lambda_{n, c}}\left(\sigma_{n, c}^{2} \sum_{k=1}^{T} \rho_{n, c}(k)+T \lambda_{n, c}^{2}\right)+T r_{n, c}^{*}(\epsilon) \lambda_{n, c} \\
& =\lambda_{n, c} E\left[Y_{n, c}(t-T)\right]-\frac{r_{n, c}^{*}(\epsilon) \sigma_{n, c}^{2}}{\lambda_{n, c}} \sum_{k=1}^{T} \rho_{n, c}(k) .
\end{aligned}
$$

Taking the expectation of (4.5) with respect to the distribution of $\vec{\Theta}(t)$ and applying the inequalities (4.6) and (4.7) yields an unconditional Lyapunov drift 
satisfying

$$
\begin{aligned}
& E[L(\vec{\Theta}(t+1))-L(\vec{\Theta}(t))]-V \sum_{n, c} E\left[U_{n, c}\left(\gamma_{n, c}(t)\right)\right] \\
& \leq N B_{2}-2 \sum_{n, c}\left(r_{n, c}^{*}(\epsilon)+\epsilon\right) E\left[Q_{n, c}(t)\right] \\
& +2 \sum_{n, c}\left\{r_{n, c}^{*}(\epsilon) E\left[Q_{n, c}(t-T)\right]\right. \\
& \left.\quad+\frac{r_{n, c}^{*}(\epsilon) \sigma_{n, c}^{2}}{\lambda_{n, c}} \sum_{k=1}^{T} \rho_{n, c}(k)+\operatorname{Tr}_{n, c}^{*}(\epsilon)\left(\mu_{\max }^{\text {in }}+\lambda_{n, c}\right)\right\} \\
& \quad-2 \sum_{n, c}\left\{r_{n, c}^{*}(\epsilon) E\left[Y_{n, c}(t-T)\right]-\left(\frac{r_{n, c}^{*}(\epsilon) \sigma_{n, c}}{\lambda_{n, c}}\right)^{2} \sum_{k=1}^{T} \rho_{n, c}(k)\right\} \\
& +2 \sum_{n, c} r_{n, c}^{*}(\epsilon) E\left[Y_{n, c}(t)\right]-V \sum_{n, c} U_{n, c}\left(r_{n, c}^{*}(\epsilon)\right) .
\end{aligned}
$$

Summing the inequalities over $t \in\{0, \ldots, M-1\}$, dividing by $M$, and taking the limit as $M \rightarrow \infty$ results in

$$
\begin{aligned}
& -V \lim _{M \rightarrow \infty} \frac{1}{M} \sum_{\tau=0}^{M-1} \sum_{n, c} E\left[U_{n, c}\left(\gamma_{n, c}(\tau)\right)\right] \\
& \leq N B_{2}-2 \epsilon \lim _{M \rightarrow \infty} \frac{1}{M} \sum_{\tau=0}^{M-1} \sum_{n, c} E\left[Q_{n, c}(\tau)\right] \\
& +2 \sum_{n, c}\left\{\left(\frac{r_{n, c}^{*}(\epsilon)}{\lambda_{n, c}}+\left(\frac{r_{n, c}^{*}(\epsilon)}{\lambda_{n, c}}\right)^{2}\right) \sigma_{n, c}^{2} \sum_{k=1}^{T} \rho_{n, c}(k)+\operatorname{Tr}_{n, c}^{*}(\epsilon)\left(\mu_{\max }^{\text {in }}+\lambda_{n, c}\right)\right\} \\
& -V \sum_{n, c} U_{n, c}\left(r_{n, c}^{*}(\epsilon)\right),
\end{aligned}
$$

where we used the fact that the system starts with empty queues, i.e., $L(\vec{\Theta}(0))=$ 0 , the nonnegativity of the Lyapunov function, the lim version of identity (3.8), and the fact that

$$
\lim _{M \rightarrow \infty} \frac{1}{M} \sum_{\tau=0}^{M-1} E\left[Y_{n, c}(\tau-T)\right]=\lim _{M \rightarrow \infty} \frac{1}{M} \sum_{\tau=0}^{M-1} E\left[Y_{n, c}(\tau)\right]
$$


Rearranging (4.9) and dividing by $2 \epsilon$, we have

$$
\begin{aligned}
& \lim _{M \rightarrow \infty} \frac{1}{M} \sum_{\tau=0}^{M-1} \sum_{n, c} E\left[Q_{n, c}(\tau)\right] \\
& \leq \frac{N B_{2}+V N U_{\max }}{2 \epsilon}+\frac{1}{\epsilon} \sum_{n, c}\left\{2 \sigma_{n, c}^{2} \sum_{k=1}^{T} \rho_{n, c}(k)+T \lambda_{n, c}\left(\mu_{\max }^{\text {in }}+\lambda_{n, c}\right)\right\}
\end{aligned}
$$

where we used the fact that $r_{n, c}^{*}(\epsilon) / \lambda_{n, c} \leq 1$ and the nonnegativity of the utility function. Rearranging (4.9) differently, dividing by $V$, and using $r_{n, c}^{*}(\epsilon) / \lambda_{n, c} \leq 1$ and the nonnegativity of queue backlogs yields

$$
\begin{aligned}
\lim _{M \rightarrow \infty} \frac{1}{M} \sum_{\tau=0}^{M-1} \sum_{n, c} E\left[U_{n, c}\left(\gamma_{n, c}(\tau)\right)\right] \geq \sum_{n, c} U_{n, c}\left(r_{n, c}^{*}(\epsilon)\right)-\frac{N B_{2}}{V} \\
-\frac{2}{V} \sum_{n, c}\left\{2 \sigma_{n, c}^{2} \sum_{k=1}^{T} \rho_{n, c}(k)+T \lambda_{n, c}\left(\mu_{\max }^{\mathrm{in}}+\lambda_{n, c}\right)\right\} .
\end{aligned}
$$

Applying Jensen's inequality based on the concavity of the utility functions, we have

$$
\frac{1}{M} \sum_{\tau=0}^{M-1} E\left[U_{n, c}\left(\gamma_{n, c}(\tau)\right)\right] \leq U_{n, c}\left(\frac{1}{M} \sum_{\tau=0}^{M-1} E\left[\gamma_{n, c}(\tau)\right]\right) .
$$

On the other hand, we can show that virtual flow queues $Y_{n, c}(t)$ are always stable under the joint flow control and backpressure policy, implying $\bar{\gamma}_{n, c}(t) \leq \bar{r}_{n, c}(t)$, which are the time averages up to slot $t$. The stability of the virtual queues can be established via a simple argument. Suppose that $Y_{n, c}(t)>\frac{1}{2} V \theta_{n, c}$, where $\theta_{n, c}$ is the bound on the first derivative of $U_{n, c}$. Then the solution to (4.3) is always $\gamma_{n, c}=0$ until $Y_{n, c}(t)$ drops below $\frac{1}{2} V \theta_{n, c}$. Suppose now that $Y_{n, c}(t) \leq \frac{1}{2} V \theta_{n, c}$. Then the virtual queue can grow at most by $A_{\text {max }}$, but after that, it cannot grow further, because $Y_{n, c}(t)$ exceeds $\frac{1}{2} V \theta_{n, c}$. Therefore, the virtual queue $Y_{n, c}(t)$ is always bounded below $\frac{1}{2} V \theta_{n, c}+A_{\max }$. Finally, noting that taking limsup or liminf instead of lim does not change the results because the inequality (4.8) holds for all $t$ and optimizing $\epsilon$ for (4.11) and (4.12) separately over $\left(0, \lambda_{\max }\right]$ completes the proof.

Theorem 4.1 shows that the joint flow control and backpressure policy stabilizes the network even when the arrival rate vector is outside the stability region and the arrival processes are time-correlated over a fixed length of interval. The theorem also shows that the joint policy yields an admission rate vector that is arbitrarily close to the utility-optimal operating point with a corresponding tradeoff in average network delay, where the parameter $V$ enables us to exploit 
the tradeoff. We now proceed to a general case in which arrivals may have memory of infinite length, such as short- and long-range dependent processes.

Theorem 4.2. If the absolute autocorrelation functions $\left|\rho_{n, c}(k)\right|$ of the arrival processes are monotonically decreasing for all $(n, c)$ pairs, then the joint flow control and backpressure policy stabilizes the network and yields bounds on the timeaverage queue backlog as

$$
\begin{aligned}
& \limsup _{t \rightarrow \infty} \frac{1}{t} \sum_{\tau=0}^{t-1} \sum_{n, c} E\left[Q_{n, c}(\tau)\right] \\
& \quad \leq \frac{1}{2\left(\lambda_{\max }-\delta\right)}\left(N B_{2}+V N U_{\max }+V \delta \sum_{n, c} \theta_{n, c}+2 \sum_{n, c} \psi_{n, c}\left(T_{\delta}\right)+2 \delta N A_{\max }\right)
\end{aligned}
$$

and network utility as

$$
\begin{aligned}
& \liminf _{t \rightarrow \infty} \sum_{n, c} U_{n, c}\left(\bar{r}_{n, c}(t)\right) \\
& \geq \sum_{n, c} U_{n, c}\left(r_{n, c}^{*}\right)-\delta \sum_{n, c} \theta_{n, c}-\frac{1}{V}\left(N B_{2}+2 \sum_{n, c} \psi_{n, c}\left(T_{\delta}\right)+2 \delta N A_{\max }\right),
\end{aligned}
$$

where $\delta$ is an arbitrary constant satisfying $0<\delta<\lambda_{\max }$ and $T_{\delta}$ is the minimum of $T$ such that $\left|E\left[A_{n, c}(t) \mid H(t-k)\right]-\lambda_{n, c}\right| \leq \delta$ for $k \geq T$ and for all $(n, c)$ pairs.

Proof. As in the proof of Theorem 4.1, the main difficulty in analyzing the drift expression in (4.5) is due to the correlations between $Q_{n, c}(t)$ and $A_{n, c}(t)$ and between $Y_{n, c}(t)$ and $A_{n, c}(t)$. Assume that $\delta$ and $T_{\delta}$ are chosen such that the conditions described in the theorem are met. Then for $T \geq T_{\delta}$, we have

$$
\begin{aligned}
& E\left[Q_{n, c}(t) A_{n, c}(t)\right] \\
& \quad \leq\left(\lambda_{n, c}+\delta\right) E\left[Q_{n, c}(t-T)\right]+\sigma_{n, c}^{2} \sum_{k=1}^{T} \rho_{n, c}(k)+T \lambda_{n, c}\left(\mu_{\max }^{\text {in }}+\lambda_{n, c}\right)
\end{aligned}
$$

and

$$
E\left[Y_{n, c}(t) A_{n, c}(t)\right] \geq\left(\lambda_{n, c}-\delta\right) E\left[Y_{n, c}(t-T)\right]-\frac{r_{n, c}^{*}(\epsilon) \sigma_{n, c}^{2}}{\lambda_{n, c}} \sum_{k=1}^{T} \rho_{n, c}(k) .
$$

Taking the expectation of (4.5) with respect to the distribution of $\vec{\Theta}(t)$ and applying the inequalities (4.13) and (4.14) yields an unconditional Lyapunov 
drift satisfying

$$
\begin{aligned}
& E[L(\vec{\Theta}(t+1))-L(\vec{\Theta}(t))]-V \sum_{n, c} E\left[U_{n, c}\left(\gamma_{n, c}(t)\right)\right] \\
& \leq N B_{2}-2 \sum_{n, c}\left(r_{n, c}^{*}(\epsilon)+\epsilon\right) E\left[Q_{n, c}(t)\right] \\
& +2 \sum_{n, c}\left\{\left(r_{n, c}^{*}(\epsilon)+\frac{r_{n, c}^{*}(\epsilon) \delta}{\lambda_{n, c}}\right) E\left[Q_{n, c}(t-T)\right]\right. \\
& \left.\quad+\frac{r_{n, c}^{*}(\epsilon) \sigma_{n, c}^{2}}{\lambda_{n, c}} \sum_{k=1}^{T} \rho_{n, c}(k)+\operatorname{Tr}_{n, c}^{*}(\epsilon)\left(\mu_{\max }^{\mathrm{in}}+\lambda_{n, c}\right)\right\} \\
& \quad-2 \sum_{n, c}\left\{\left(r_{n, c}^{*}(\epsilon)-\frac{r_{n, c}^{*}(\epsilon) \delta}{\lambda_{n, c}}\right) E\left[Y_{n, c}(t-T)\right]-\left(\frac{r_{n, c}^{*}(\epsilon) \sigma_{n, c}}{\lambda_{n, c}}\right)^{2} \sum_{k=1}^{T} \rho_{n, c}(k)\right\} \\
& +2 \sum_{n, c} r_{n, c}^{*}(\epsilon) E\left[Y_{n, c}(t)\right]-V \sum_{n, c} U_{n, c}\left(r_{n, c}^{*}(\epsilon)\right) .
\end{aligned}
$$

Summing the inequalities over time slots $t \in\{0, \ldots, M-1\}$, dividing by $M$, and taking the limit as $M \rightarrow \infty$ results in

$$
\begin{aligned}
& -V \lim _{M \rightarrow \infty} \frac{1}{M} \sum_{\tau=0}^{M-1} \sum_{n, c} E\left[U_{n, c}\left(\gamma_{n, c}(\tau)\right)\right] \\
& \leq N B_{2}-2(\epsilon-\delta) \lim _{M \rightarrow \infty} \frac{1}{M} \sum_{\tau=0}^{M-1} \sum_{n, c} E\left[Q_{n, c}(\tau)\right] \\
& \quad+2 \delta \lim _{M \rightarrow \infty} \frac{1}{M} \sum_{\tau=0}^{M-1} \sum_{n, c} E\left[Y_{n, c}(\tau)\right] \\
& +2 \sum_{n, c}\left\{2 \sigma_{n, c}^{2} \sum_{k=1}^{T} \rho_{n, c}(k)+T \lambda_{n, c}\left(\mu_{\max }^{\text {in }}+\lambda_{n, c}\right)\right\} \\
& -V \sum_{n, c} U_{n, c}\left(r_{n, c}^{*}(\epsilon)\right)
\end{aligned}
$$

where we used the fact that the system starts with empty queues, $r_{n, c}^{*}(\epsilon) / \lambda_{n, c} \leq$ 1 , the nonnegativity of the Lyapunov function, and the lim versions of identities (3.8) and (4.10). Rearranging terms, dividing by $2(\epsilon-\delta)$, and using the 
nonnegativity of the utility function, we obtain

$$
\begin{aligned}
\lim _{M \rightarrow \infty} & \frac{1}{M} \sum_{\tau=0}^{M-1} \sum_{n, c} E\left[Q_{n, c}(\tau)\right] \\
\leq & \frac{N B_{2}+V N U_{\max }}{2(\epsilon-\delta)}+\frac{1}{\epsilon-\delta} \sum_{n, c}\left\{2 \sigma_{n, c}^{2} \sum_{k=1}^{T} \rho_{n, c}(k)+T \lambda_{n, c}\left(\mu_{\max }^{\text {in }}+\lambda_{n, c}\right)\right\} \\
& +\frac{\delta}{\epsilon-\delta} \lim _{M \rightarrow \infty} \frac{1}{M} \sum_{\tau=0}^{M-1} \sum_{n, c} E\left[Y_{n, c}(\tau)\right] .
\end{aligned}
$$

Rearranging (4.16) differently, dividing by $V$, and using the nonnegativity of the queue backlogs, we have

$$
\begin{aligned}
& \lim _{M \rightarrow \infty} \frac{1}{M} \sum_{\tau=0}^{M-1} \sum_{n, c} E\left[U_{n, c}\left(\gamma_{n, c}(\tau)\right)\right] \\
& \geq \sum_{n, c} U_{n, c}\left(r_{n, c}^{*}(\epsilon)\right)-\frac{N B_{2}}{V}-\frac{2}{V} \sum_{n, c}\left\{2 \sigma_{n, c}^{2} \sum_{k=1}^{T} \rho_{n, c}(k)+T \lambda_{n, c}\left(\mu_{\max }^{\mathrm{in}}+\lambda_{n, c}\right)\right\} \\
& \quad-\frac{2 \delta}{V} \lim _{M \rightarrow \infty} \frac{1}{M} \sum_{\tau=0}^{M-1} \sum_{n, c} E\left[Y_{n, c}(\tau)\right] .
\end{aligned}
$$

The rest of the proof follows closely that of Theorem 4.1, and the result is obtained by optimizing $\epsilon$ for (4.17) and (4.18) separately over $\left(0, \lambda_{\max }\right]$ and using the fact that $Y_{n, c}(t)$ is always bounded below $\frac{1}{2} V \theta_{n, c}+A_{\max }$ from the proof of Theorem 4.1. Finally, note that because inequality (4.15) holds for all $t$, taking limsup or liminf instead of lim does not change the results.

In the case of i.i.d. arrivals, it was shown that the sum of average queue backlogs is at most $\left(N B_{2}+V N U_{\max }\right) / 2 \lambda_{\max }$ and the achieved network utility is at worst $N B_{2} / V$ apart from the value at the utility-optimal operating point [Neely et al. 05a]. Therefore, the additional terms in the tradeoff of network utility and sum of average queue backlog in Theorems 4.1 and 4.2 can be viewed as a penalty due to the correlations on the arrival processes. In Theorem 4.1, we needed to increase $V$ to achieve performance that is arbitrarily close to that of the optimal utility value with a corresponding increase in the sum of average queue backlogs. In the case of Theorem 4.2, as $V$ goes to infinity, the achieved network utility approaches a point that is at $\operatorname{most} \delta \sum_{n, c} \theta_{n, c}$ from the optimal network utility. 


\section{Concluding Remarks}

The performance of backpressure-based stochastic network controls in multihop wireless networks with time-correlated arrivals was studied using the Lyapunov drift technique. The impact of input correlations is reflected in the upper bound on the average network delay when the arrival rate vector is inside the stability region and the tradeoff between the average network delay and utility when the arrival rate vector is possibly outside the stability region. To handle the Lyapunov drift with correlated terms, we first expressed the evolution of the system dynamics over multiple slots and used the fact that the conditional expectation of the arrival process given the past history falls within arbitrarily small constant range around its unconditional expectation if the time-lag between the arrival process and the past history becomes sufficiently large. This is true for both finite memory and infinite memory arrivals with monotonically decreasing autocorrelation functions. On the other hand, a usual sum-of-queue-squares-type Lyapunov function was used in which no cross-terms between different links are emerging. Therefore, in order to incorporate the explicit form of the cross-correlation between the different links, one must modify the Lyapunov function.

Acknowledgments. The material in this paper was presented in part at the Information Theory and Applications Workshop, San Diego, California, February 2011 and the 9th International Symposium on Modeling and Optimization in Mobile, Ad Hoc, and Wireless Networks, Princeton, New Jersey, May 2011. This work was supported by MURI grant W911NF-08-1-0238, NSF grant CCF-0728966, ONR grant N000141110127, and NSF grant CCF-0905209. J. Jeon was partially supported by the NIST-ARRA Fellowship Program.

\section{References}

[Asmussen 03] S. Asmussen. Applied Probability and Queues. Springer, 2003.

[Beran et al. 95] J. Beran, R. Sherman, M. S. Taqqu, and W. Willinger. "Long-Range Dependence in Viriable-Bit-Rate Video Traffic." IEEE Transactions on Communications 43:2-4 (1995), 1566-1579.

[Bertsekas and Gallager 92] D. Bertsekas and R. Gallager. Data Networks. Prentice Hall, 1992.

[Chiang 05] M. Chiang. "Balancing Transport and Physical Layers in Wireless Multihop Networks: Jointly Optimal Congestion Control and Power Control." IEEE Journal on Selected Areas in Communications 23:1 (2005), 104-116.

[Crovella and Bestavros 97] M. E. Crovella and A. Bestavros. "Self-Similarity in World Wide Web Traffic: Evidence and Possible Causes." IEEE/ACM Transactions on Networking 5:6 (1997), 835-846. 
[Georgiadis et al. 06] L. Georgiadis, M. J. Neely, and L. Tassiulas. "Resource Allocation and Cross-Layer Control in Wireless Networks." Foundations and Trends in Networking 1:1 (2006), 1-149.

[Kelly 97] F. Kelly. "Charging and Rate Control for Elastic Traffic." European Transactions on Telecommunications 8 (1997), 33-37.

[Kelly et al. 98] F. Kelly, A. K. Maulloo, and D. K. H. Tan. "Rate Control for Communication Networks: Shadow Prices, Proportional Fairness and Stability." Journal of the Operational Research Society 49:3 (1998), 237-252.

[Le et al. 09] L. B. Le, K. Jagannathan, and E. Modiano. "Delay Analysis of Maximum Weight Scheduling in Wireless Ad Hoc Networks." In Proceedings of Conference on Information Sciences and Systems (CISS), pp. 389-394, 2009.

[Le et al. 10] L. B. Le, E. Modiano, and N. B. Shroff. "Optimal Control of Wireless Networks with Finite Buffers." In Proceedings of IEEE INFOCOM, pp. 1316-1329, 2010 .

[Leland et al. 94] W. Leland, M. Taqqu, W. Willinger, and D. Wilson. "On the SelfSimilar Nature of Ethernet Traffic (Extended Version)." IEEE/ACM Transactions on Networking 2 (1994), 1-15.

[Low and Lapsley 99] S. H. Low and D. E. Lapsley. "Optimization Flow Control-1: Basic Algorithm and Convergence." IEEE/ACM Transactions on Networking 7:6 (1999), 861-874.

[Mekkittikul and McKeown 98] A. Mekkittikul and N. McKeown. "A Practical Scheduling Algorithm to Achieve 100\% Throughput in Input-Queued Switches." In Proceedings of IEEE INFOCOM, pp. 792-799, 1998.

[Mo and Walrand 00] J. Mo and J. Walrand. "Fair End-to-End Window-Based Congestion Control." IEEE/ACM Transactions on Networking 8:5 (2000), 556-567.

[Neely 06a] M. J. Neely. "Energy Optimal Control for Time Varying Wireless Networks." IEEE Transactions on Information Theory 52:7 (2006) 2915-2934.

[Neely 06b] M. J. Neely. "Super-Fast Delay Tradeoffs for Utility Optimal Fair Scheduling in Wireless Networks." IEEE Journal on Selected Areas in Communications 24:8 (2006), 1489-1501.

[Neely 06c] M. J. Neely. "Order Optimal Delay for Opportunistic Scheduling In Multiuser Wireless Uplinks and Downlinks." In Proceedings of the 44th Annual Allerton Conference on Communication, Control, and Computing, pp. 67-76, September 2006.

[Neely 09] M. J. Neely. "Delay Analysis for Maximal Scheduling with Flow Control in Wireless Networks with Bursty Traffic." IEEE/ACM Transactions on Networking 17:4 (2009), 1146-1159.

[Neely et al. 05a] M. J. Neely, E. Modiano, and C.-P. Li. "Fairness and Optimal Stochastic Control for Heterogeneous Networks." In Proceedings of IEEE INFOCOM, pp. 1723-1734, March 2005.

[Neely et al. 05b] M. J. Neely, E. Modiano, and C. E. Rohrs. "Dynamic Power Allocation and Routing for Time-Varying Wireless Networks." IEEE Journal on Selected Areas in Communications 23:1 (2005), 89-103. 
[Paxson and Floyd 95] V. Paxson and S. Floyd. "Wide-Area Traffic: The Failure of Poisson Modeling." IEEE/ACM Transactions on Networking 3:3 (1995), 226-244.

[Samorodnitsky 07] G. Samorodnitsky. "Long Range Dependence." Foundations and Trends in Stochastic Systems 1:3 (2007), 163-257.

[Tassiulas 97] L. Tassiulas. "Scheduling and Performance Limits of Networks with Constantly Changing Topology." IEEE Transactions on Information Theory 43:3 (1997), 1067-1073.

[Tassiulas and Ephremides 92] L. Tassiulas and A. Ephremides. "Stability Properties Of Constrained Queueing Systems and Scheduling Policies for Maximum Throughput in Multihop Radio Networks." IEEE Transactions on Automatic Control 37:12 (1992), 1936-1948.

Jeongho Jeon, Department of Electrical and Computer Engineering and Institute for Systems Research, University of Maryland, College Park, MD 20742 (jeongho@umd.edu)

Anthony Ephremides, Department of Electrical and Computer Engineering and Institute for Systems Research, University of Maryland, College Park, MD 20742 (etony@umd.edu) 